\title{
A Review of Aceclofenac: Analgesic and Anti-Inflammatory Effects on Musculoskeletal Disorders
}

\author{
Giovanni lolascon (iD) \\ Sergio Giménez ${ }^{2}$ \\ Dorottya Mogyorósi, ${ }^{3,4}$ \\ 'Department of Medical and Surgical \\ Specialties and Dentistry, University of \\ Campania "Luigi Vanvitelli", Naples, Italy; \\ ${ }^{2}$ Family Medicine, UGC Limonar, Malaga, \\ Spain; ${ }^{3}$ State Medical Center of \\ Szentendre, Budapest, Hungary; ${ }^{4}$ Buda \\ Health Center, Budapest, Hungary
}

Correspondence: Giovanni lolascon Department of Medical and Surgical Specialties and Dentistry, University of Campania “Luigi Vanvitelli”, Naples, Italy Email giovanni.iolascon@gmail.com

\begin{abstract}
Aceclofenac is an oral non-steroidal anti-inflammatory drug (NSAID) with antiinflammatory and analgesic properties. Although there are some differences in the authorized indications between countries, aceclofenac is mainly recommended for the treatment of inflammatory and painful processes, such as low back pain (LBP), scapulohumeral periarthritis, extraarticular rheumatism, odontalgia, and osteoarthritis (OA), rheumatoid arthritis (RA), and ankylosing spondylitis (AS). The analgesic properties and tolerability profile of aceclofenac in musculoskeletal disorders are reviewed, focusing on relevant and recent studies. The efficacy and safety comparison of aceclofenac with other analgesics and anti-inflammatory agents in OA, AS, RA, and LBP is described. Relevant studies were identified following a literature search of PubMed using the terms "aceclofenac" and "clinical trials" published from 1 Jan 1992 to 1 Jan 2020. Aceclofenac is at least as effective as other NSAIDs in reducing pain and/or improving functional capacity in chronic pain conditions (OA, AS, RA, and LBP). It is generally well tolerated and appears to have a more favorable GI profile than other NSAIDs. Thus, current evidence indicates that aceclofenac is a useful option for the management of pain and inflammation across a wide range of painful conditions.
\end{abstract}

Keywords: aceclofenac, NSAIDs, analgesia, osteoarthritis, low back pain

\section{Introduction}

Aceclofenac is a phenylacetic acid derivative nonsteroidal anti-inflammatory drug (NSAID) with marked anti-inflammatory and analgesic properties. ${ }^{1,2}$ It is a potent inhibitor of cyclooxygenase (COX), ${ }^{1,3,4}$ a key enzyme in the synthesis of prostaglandins and thromboxanes with selectivity for the COX-2 over COX-1 isoform. ${ }^{1}$ Aceclofenac was first approved in the EU in 1990 and launched in Spain in 1992. Since then, it has been approved for use in 69 countries worldwide and has an estimated exposure of about 171 million patients treated. The authorized indications of aceclofenac vary between countries, but in general, it is recommended for the treatment of inflammatory and painful processes, such as low back pain (LBP), odontalgia, scapulohumeral periarthritis, and extraarticular rheumatism, as well as for the treatment of osteoarthritis (OA), rheumatoid arthritis (RA), and ankylosing spondylitis (AS). ${ }^{4}$ The pharmacological properties, efficacy and tolerability of aceclofenac have been reviewed previously elsewhere. ${ }^{1,2}$ This article reviews the comparative efficacy and tolerability of aceclofenac $100 \mathrm{mg}$ twice daily monotherapy in musculoskeletal disorders, including OA, RA, AS, and LBP, focusing on relevant and more recent studies where available. 


\section{Pharmacology}

The main signs and symptoms of inflammation are related to the synthesis and physiological action of prostaglandins (eg, PGE2) in damaged tissue, which cause vasodilation (redness, heat, swelling), inflammation and pain. ${ }^{5}$ The dysregulation of synthesis or degradation of PGE2, one of the most abundant prostaglandins, has been associated with processes leading to symptoms of inflammation. PGE2-mediated increases in arterial dilation and microvascular permeability result in increased blood flow into the inflamed tissue causing redness and oedema. The action of PGE2 on peripheral sensory neurons and central sites within the spinal cord and brain result in pain. ${ }^{5}$ In inflamed joints, PGE2 induces bone resorption by osteoclasts. $^{6}$

Prostaglandins are produced by COX, an enzyme with two major isoforms, COX-1 and COX-2. COX-1 is constitutively expressed in almost all tissues and is responsible for the production of prostaglandins for homeostatic functions (eg protective effect on gastric mucosa). ${ }^{7} \mathrm{COX}-2$ is induced by inflammatory mediators (like cytokines, growth factors and bacterial endotoxins) and increases the production of prostaglandins that mediate inflammation, pain, and fever. ${ }^{7}$

The mechanism of action of aceclofenac is largely based on the inhibition of prostaglandin production by selectively inhibiting COX-2 (half maximal inhibitory concentration $\left[\mathrm{IC}_{50}\right] \mathrm{COX}-2 / \mathrm{COX}-1$ ratio 0.26$) .{ }^{1}$ In clinical studies, aceclofenac inhibited prostaglandin synthesis in synovial fluid from patients with acute knee OA and in peripheral blood leukocytes from patients with OA. ${ }^{1}$ NSAID treatment with aceclofenac (100 mg twice daily) for 3 months inhibited COX-2 synthesis in the knee synovial membrane of 30 patients with OA scheduled for total knee replacement surgery versus patients with OA who did not want to be treated with NSAIDs (control group). Results showed that aceclofenac reduced PGE2 in the synovial fluid ( $p<0.05 \mathrm{vs}$ control), and protein expression $\left(p<0.05\right.$ vs control) at the synovial membrane. ${ }^{8}$ In another study in 30 patients with OA scheduled for knee replacement surgery, 3-months' treatment with aceclofenac decreased IL-1 $\beta$-induced release of PGE2 and decreased the synthesis of COX-2 and microsomal prostaglandin E synthase (mPGES)-1 in the cartilage and chondrocytes. In addition, aceclofenac reduced IL-1 $\beta$-induced expression of TNF $\alpha$ and IL-1 $\beta$ in cultured OA chondrocytes. ${ }^{9}$ Aceclofenac also reduced lymphocyte adhesion in in vitro studies and increased glycosaminoglycan production in cartilage from OA patients. ${ }^{1,2}$

Oral aceclofenac is rapidly and completely absorbed, with peak plasma concentrations reached approximately 1.25 to $3.0 \mathrm{~h}$ after dosing. ${ }^{1,3}$ After penetrating into the synovial fluid, aceclofenac concentrations reach approximately $57 \%$ of those in the plasma. Aceclofenac is highly $(>99 \%)$ protein bound and has a volume of distribution of approximately $25 \mathrm{~L}$. Aceclofenac circulates mainly as unchanged drug. Aceclofenac is metabolized into its main metabolite 4'-hydroxyaceclofenac and several minor metabolites, including 5-hydroxyaceclofenac, diclofenac, 4'-hydroxydiclofenac. Approximately $75 \%$ of an administered aceclofenac dose is excreted via urine, largely as hydroxymetabolites. Aceclofenac mean elimination halflife is approximately $4 \mathrm{~h} .^{1,3}$

Some studies suggest that diclofenac and other metabolites of aceclofenac may cause toxicity in aquatic and terrestrial organisms, such as mussels and Gyps vultures. ${ }^{10,11}$ However, data currently available are inconclusive and additional studies are required to better assess the fate and toxic effects of aceclofenac and its metabolites. It has also been suggested that wastewater treatment plants should be equipped with more advanced treatment methods to more effectively remove potential contaminants, such as aceclofenac metabolites. ${ }^{10}$

\section{Efficacy Profile}

Rheumatic or musculoskeletal conditions consist of over 150 progressive painful diseases and syndromes that can be broadly categorized as joint diseases, physical disability, spinal disorders, and conditions resulting from trauma. ${ }^{12}$ Musculoskeletal diseases are very common, with an estimated global prevalence of $11.9 \%$ for $\mathrm{LBP}^{13} 3.8 \%$ for knee ${ }^{13},{ }^{14} 0.85 \%$ for hip $\mathrm{OA},{ }^{14}$ $0.24 \%$ for $\mathrm{RA}^{15}$ and $0.18 \%$ for AS. ${ }^{16}$ Musculoskeletal conditions are one of the leading causes of morbidity and disability, placing a heavy burden on healthcare costs and resulting in loss of work. ${ }^{12,17}$ In 2016, musculoskeletal disorders accounted for 140 million total disabilityadjusted life years (DALY; the sum of years of life lost due to premature mortality and years lived with disability), with LBP, neck pain, OA, and RA accounting for 57.6, 28.9, 16.2, and 5.5 million DALYs, respectively. ${ }^{18}$

As reviewed in previous articles, numerous randomized studies have demonstrated the efficacy of aceclofenac in reducing pain and improving functional capacity in patients with musculoskeletal disorders, including OA, 
RA, and AS; in the early phases of development, aceclofenac was shown to be superior to placebo. ${ }^{1,2}$ Results from key trials assessing the efficacy of aceclofenac versus other analgesics are reviewed in this section, with the focus on more recent data where available.

\section{Osteoarthritis}

Several randomized clinical studies of up to 12 weeks' duration showed that aceclofenac $100 \mathrm{mg}$ twice daily was generally as effective as diclofenac, nabumetone, naproxen, and piroxicam, and more effective than paracetamol in reducing pain and symptom severity and improving functional capacity in adults (aged $\geq 18$ years) with radiologically confirmed OA (see Table 1 for dosage details).

Following 4-12 weeks treatment, pain intensity (as assessed by pain scores) was reduced from baseline with aceclofenac and comparators (diclofenac, nabumetone, naproxen, piroxicam, and paracetamol) (Table 1), with the reduction in aceclofenac and piroxicam recipients seen as early as 15 days of treatment. ${ }^{19,20}$ The improvement in pain intensity was similar between treatment groups in all studies, apart from a 6-week study where results favored aceclofenac over paracetamol ${ }^{21}$ and a 4-week study with results favoring nabumetone over aceclofenac. ${ }^{22}$ Physician's assessment of pain intensity in two studies also showed no significant differences among patients receiving aceclofenac, diclofenac or naproxen, with $60-80 \%$ of patients experiencing improved pain intensity. ${ }^{23,24}$ However, unlike physician's assessment, patient's assessment of pain intensity in one study favored aceclofenac over diclofenac, with $71 \%$ versus $59 \%$ of patients experiencing a reduction in pain $(p=0.005) .{ }^{23}$

Treatment with aceclofenac reduced joint tenderness, swelling, and erythema, and improved knee flexion and extension in patients with OA (Table 2). Apart from two 8 -week studies where aceclofenac was more effective than diclofenac in reducing joint tenderness (both $\mathrm{p}<0.05$ ), ${ }^{25,26}$ no significant differences in these outcomes were seen between patients receiving aceclofenac and those receiving diclofenac, naproxen, or piroxicam (Table 2). Aceclofenac also improved functional capacity, as assessed by the Western Ontario and McMaster (WOMAC) university index and disease status scores, and improved disease severity, as assessed by several measures including Gonarthrosis Severity Index (GSI) and Osteoarthritis Severity Index (OSI), and patients' and physicians' global assessment of disease severity (Table 2). Apart from three studies where aceclofenac was more effective than comparators (diclofenac and paracetamol) in improving disease severity and functional capacity, ${ }^{21,25,26}$ there were no significant differences between treatment groups for these parameters (Table 2). Two studies showed that aceclofenac and piroxicam significantly $(p<0.05)$ improved knee flexion and knee extension within 30 days, and disease severity (GSI/OSI scores) by day $15 .{ }^{19,20}$

Consistent results were seen in a small randomized, double-blind, single-center study ( $\mathrm{n}=60$ patients) comparing aceclofenac $100 \mathrm{mg}$ twice daily with diclofenac $50 \mathrm{mg}$ three times daily, which found no significant differences between the two treatment groups at week 8 in terms of the reduction in pain intensity on movement; joint tenderness, swelling and redness; and functional capacity. ${ }^{27}$

Furthermore, a meta-analysis of eight double-blind studies ${ }^{19-21-23-25-27,28}$ and one single-blind study ${ }^{26}$ compared the efficacy of aceclofenac with that of comparators in terms of pain reduction (meta-analysis of seven studies) and improving physical function (meta-analysis of 8 studies). ${ }^{29}$ Results found no significant difference between aceclofenac and comparators in pain reduction (standardized mean difference $[\mathrm{SDM}]-3.0 ; 95 \% \mathrm{CI}-0.62,0.01$ ) and found a significant difference in favor of aceclofenac in improving physical function (SDM $-0.27 ; 95 \% \mathrm{CI}-0.50$, -0.03 ); however, it should be noted that there was significant $(p=0.0001)$ heterogeneity between trials for both pain reduction $\left(\mathrm{I}^{2}=88 \%\right)$ and physical function $\left(\mathrm{I}^{2}=81 \%\right)$, which limits the interpretation of results. ${ }^{29}$

\section{Ankylosing Spondylitis}

Aceclofenac $100 \mathrm{mg}$ twice daily was at least as effective as indomethacin, naproxen, and tenoxicam in reducing pain and morning stiffness and improving mobility in three 12-week, randomized clinical trials in patients with AS (see Table 3 for dosage details). Following 12 weeks' treatment, pain intensity (as assessed by pain scores) was significantly $(p<0.01)$ reduced from baseline in patients receiving aceclofenac or comparators (indomethacin, naproxen, and tenoxicam), with no significant between-group differences (Table 3). ${ }^{30-32}$ Treatment with aceclofenac and comparators also significantly $(p<0.05)$ improved the duration of morning stiffness (where assessed) and spinal mobility (assessed by modified Schöber's test, the C7 to iliac crest, hand-to-floor distance measurement, lateral spinal flexion, chest expansion, and/or the occiput-to-wall distance), ${ }^{30-32}$ apart from C7 to iliac crest distance with aceclofenac in the comparison with 
Table I Analgesic Efficacy of Aceclofenac in Patients with Osteoarthritis

\begin{tabular}{|c|c|c|c|}
\hline \multirow[t]{2}{*}{ Study (Design) } & \multirow{2}{*}{$\begin{array}{l}\text { Treatment } \\
\text { (Duration) }\end{array}$} & \multirow{2}{*}{$\begin{array}{l}\text { ITT No. } \\
\text { of pts }\end{array}$} & \multirow{2}{*}{$\begin{array}{l}\text { Pain Intensity (ACE vs Comparator) } \\
\text { Mean Values }{ }^{\text {a, b }}\end{array}$} \\
\hline & & & \\
\hline \multicolumn{4}{|l|}{ Versus diclofenac } \\
\hline $\begin{array}{l}\text { Ward et al. } 1995^{23} \\
(\mathrm{r}, \mathrm{db}, \mathrm{mc})\end{array}$ & $\begin{array}{l}\text { ACE vs DIC (12 } \\
\text { weeks) }\end{array}$ & 200 vs 197 & $\begin{array}{l}\text { Both groups had reduced joint pain }{ }^{c}(P E) \text { at rest ( } p=0.000 \text { I vs BL) and on movement; no } \\
\text { significant difference in } \% \text { of patients with reduced pain intensity at rest }(75 \text { vs } 70 \%) \text { and } \\
\text { on movement }(70 \% \text { vs } 63 \%)\end{array}$ \\
\hline $\begin{array}{l}\text { Pareek et al. } \\
2006^{25} \mathrm{~d}(\mathrm{r}, \mathrm{db}, \mathrm{mc})\end{array}$ & $\begin{array}{l}\text { ACE vs DIC }(8 \\
\text { weeks })\end{array}$ & 125 vs 122 & $\begin{array}{l}\text { VAS score }(P E) \text { decreased from } B L \text { in both groups: right knee on weight bearing ( } 2.4 \text { vs } \\
3.4 ; \mathrm{BL} \approx 5) \text {; at rest }(1.9 \text { vs } 2.9 ; \mathrm{BL} \approx 4) \text {; on active movement }(2.5 \text { vs } 3.5 ; \mathrm{BL} \approx 5)\end{array}$ \\
\hline $\begin{array}{l}\text { Patil et al. } 2012^{26 d} \\
(r, s b, s c)\end{array}$ & $\begin{array}{l}\text { ACE vs DIC ( } 8 \\
\text { weeks) }\end{array}$ & 60 vs 58 & $\begin{array}{l}\text { VAS score decreased from } B L \text { in both groups: on weight bearing ( } 2.8 \text { vs } 2.9 ; B L \approx 5) \text {; at rest } \\
(2.4 \text { vs } 2.7 ; B L \approx 5) \text {; on active movement }(2.9 \text { vs } 3.7 ; B L \approx 5) \text {; all } p<0.000 \text { I vs } B L\end{array}$ \\
\hline $\begin{array}{l}\text { Pareek et al. } \\
2013^{28}(\mathrm{r}, \mathrm{db}, \mathrm{mc})\end{array}$ & $\begin{array}{l}\text { ACE vs DIC }(6 \\
\text { weeks })\end{array}$ & 284 vs 285 & VAS score decreased from BL in both groups (mean change -2.7 vs -2.8 ) \\
\hline \multicolumn{4}{|l|}{ Vs nabumetone } \\
\hline $\begin{array}{l}\text { Gijón-Baños et al } \\
1997^{54} \text { (r, ol, mc) }\end{array}$ & $\begin{array}{l}\text { ACE vs NAB (I } 2 \\
\text { weeks) }\end{array}$ & 137 vs 137 & VAS score $(P E)$ decreased from $B L$ in both groups ( 4.3 vs $4.4 ; B L 6.1$ ); both $p<0.00$ I vs $B L$ \\
\hline $\begin{array}{l}\text { Paul et al. } 2009^{22 d} \\
(r, d b, s c)\end{array}$ & $\begin{array}{l}\text { ACE vs NAB vs } \\
\text { PL (4 weeks) }\end{array}$ & $\begin{array}{l}141^{\mathrm{e}} \mathrm{vs} \\
141^{\mathrm{e}} \mathrm{vs} \\
141^{\mathrm{e}}\end{array}$ & $\begin{array}{l}\text { VAS score decreased from } B L \text { in both groups ( } 3.79 \text { vs } 4.23 \text { vs } 0.79) \text {, with a significant } \\
\text { difference in favor of } N A B(p<0.001 \text { vs } A C E)^{f}\end{array}$ \\
\hline $\begin{array}{l}\text { Jagadeesh et al. } \\
2018^{55 \mathrm{~d}} \text { (r, ol, sc) }\end{array}$ & $\begin{array}{l}\text { ACE vs NAB (12 } \\
\text { weeks) }\end{array}$ & 34 vs 34 & VAS score decreased from $B L$ in both groups ( 2.34 vs $2.37 ; B L \approx 6)$ \\
\hline \multicolumn{4}{|l|}{ Vs naproxen } \\
\hline $\begin{array}{l}\text { Kornasoff et al. } \\
1997^{24}(r, d b, m c)\end{array}$ & $\begin{array}{l}\text { ACE vs NAP (I2 } \\
\text { weeks) }\end{array}$ & 190 vs 184 & $\begin{array}{l}\text { No significant difference in } \% \text { of patients with reduced pain intensity }{ }^{c} \text { at rest ( } 76 \% \text { vs } 81 \% \text { ) } \\
\text { and on movement ( } 83 \% \text { vs } 86 \%)\end{array}$ \\
\hline \multicolumn{4}{|l|}{ Vs piroxicam } \\
\hline $\begin{array}{l}\text { Torri et al. } 1994^{20} \\
(\mathrm{r}, \mathrm{db})\end{array}$ & $\begin{array}{l}\text { ACE vs PIR (12 } \\
\text { weeks) }\end{array}$ & $89^{\mathrm{e}}$ vs $90^{\mathrm{e}}$ & VAS score decreased from $B L$ in both groups ( 23 vs $20 ; B L \approx 59$ ); both $p<0.01$ vs $B L$ \\
\hline $\begin{array}{l}\text { Perez-Busquier et al } \\
1997^{19}(\mathrm{r}, \mathrm{db}, \mathrm{mc})\end{array}$ & $\begin{array}{l}\text { ACE vs PIR }(8 \\
\text { weeks) }\end{array}$ & $\begin{array}{l}109^{\mathrm{e}} \text { vs } \\
108^{\mathrm{e}}\end{array}$ & VAS score decreased from $B L$ in both groups ( 36 vs $37 ; B L \approx 71$ ); both $p<0.001$ vs $B L$ \\
\hline \multicolumn{4}{|l|}{ Vs paracetamol } \\
\hline $\begin{array}{l}\text { Batlle-Gualda et al } \\
2007^{21}(r, d b, m c)\end{array}$ & $\begin{array}{l}\text { ACE vs PAR }(6 \\
\text { weeks) }\end{array}$ & 86 vs 82 & $\begin{array}{l}\text { VAS score }(P E) \text { decreased from } B L \text { in both groups (mean change }-18 \text { vs }-11 ; B L \approx 62 \text { ); } \\
\text { both } p<0.00 I \text { vs } B L \text { and } p=0.037 \text { vs } \mathbf{P A R}^{\mathbf{f}}\end{array}$ \\
\hline
\end{tabular}

Notes: ${ }^{a}$ Lower scores indicate improvement. ${ }^{b}$ VAS score assessed on a 0-10 mm scale, apart from three studies which used 0-100 mm scale ${ }^{19-2 !}$. ${ }^{\mathrm{A}}$ Assessed on a 5-point scale, with lower scores indicating fewer symptoms. ${ }^{d}$ Studies conducted in Indian patients. ${ }^{\mathrm{e}}$ Modified intent-to-treat ${ }^{28}$ or evaluable population ${ }^{19,20,22 f}$ Statistically significant differences between treatment groups are bolded.

Abbreviations: ACE, aceclofenac $100 \mathrm{mg}$ twice daily; BL, baseline; ACE/PAR, aceclofenac $100 \mathrm{mg} /$ paracetamol $500 \mathrm{mg}$ fixed-dose combination twice daily; db, double-blind; $\mathrm{DIC}$, diclofenac $50 \mathrm{mg}$ three times daily ${ }^{23,27,28}$ or $75 \mathrm{mg}$ twice daily; ${ }^{25,26} \mathrm{GSI}$, Gonarthrosis Severity Index; mc, multicenter; NAB, nabumetone I-2 g daily, ${ }^{54}$ I g once daily ${ }^{55}$ or 750 mg twice daily; ${ }^{22}$ NAP, naproxen 500 mg twice daily; NS, nonsignificant; ol, open label; OSI, Lequesne Osteoarthritis Severity Index; PAR, paracetamol I g three times daily; PE, primary endpoint; PGA, patient's global assessment; PhGA, physician's global assessment; PID, pain intensity difference; PIR, piroxicam 20 mg once daily; PL, placebo; pts, patients; r, randomized; sb, single-blind; sc, single center; SPID, sum of pain intensity difference.

tenoxicam. ${ }^{32}$ No significant differences between treatment groups were seen for these outcomes in all three studies. ${ }^{30-32}$

In addition, a small $(\mathrm{n}=60), 12$-week, randomized, double-blind, single-center study found that aceclofenac $100 \mathrm{mg}$ twice daily plus adjunctive tramadol/paracetamol
$37.5 \mathrm{mg} / 325 \mathrm{mg}$ fixed-dose combination twice daily was effective in reducing pain and disease activity in patients with AS, providing marginal benefits over aceclofenac monotherapy. ${ }^{33}$ At week $12,53 \%$ of patients receiving aceclofenac plus adjunctive tramadol/paracetamol compared 
Table 2 Effect of Aceclofenac on Other Outcomes in Patients with Osteoarthritis

\begin{tabular}{|c|c|c|}
\hline \multirow[t]{2}{*}{ Study (Design) } & \multirow{2}{*}{$\begin{array}{l}\text { Treatment } \\
\text { (Duration) }\end{array}$} & Other Efficacy Outcomes (ACE vs Comparator) \\
\hline & & (Mean Values Reported) \\
\hline \multicolumn{3}{|l|}{ Versus diclofenac } \\
\hline $\begin{array}{l}\text { Ward et al. } 1995^{23}(r, d b \text {, } \\
\text { mc) }\end{array}$ & $\begin{array}{l}\text { ACE vs DIC (12 } \\
\text { weeks) }\end{array}$ & $\begin{array}{l}\text { Both groups had improved: } \\
\text { - joint tenderness, swelling, erythema, functional capacity and overall assessment; all } p=N S \text { vs DIC } \\
\text { - knee flexion }\left(10^{\circ} \text { vs } 1^{\circ}\right) ; p<0.05 \text { vs BL }\end{array}$ \\
\hline Pareek et al. $2006^{25}(\mathrm{r}, \mathrm{db})$ & $\begin{array}{l}\text { ACE vs DIC }(8 \\
\text { weeks })\end{array}$ & 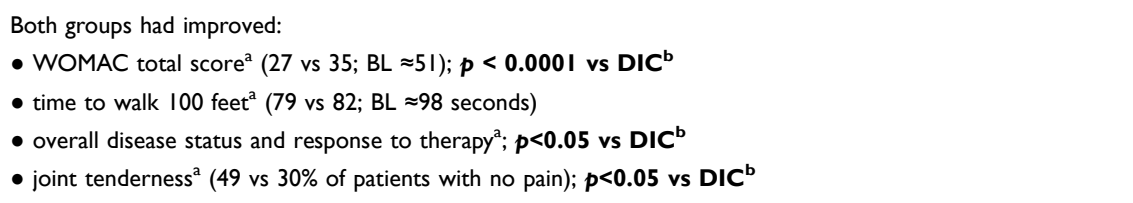 \\
\hline Patil et al. $2012^{26}(r, s b)$ & $\begin{array}{l}\text { ACE vs DIC }(8 \\
\text { weeks) }\end{array}$ & $\begin{array}{l}\text { Both groups had improved: } \\
\text { - WOMAC total score ( } 38 \text { vs } 43 ; B L \approx 57) ; p<0.000 \text { I vs } B L ; p<0.000 \text { I vs DIC } \\
\text { - time to walk } 100 \text { feet ( } 83 \text { vs } 85 ; B L \approx 102 \text { seconds); } p<0.000 \text { I vs } B L \text { disease status score }(2.8 \text { vs } 2.5 ; B L \approx 2.3 \text { ); } \\
\text { p }<<0.05 \text { vs } B L ; p<0.05 \text { vs DIC } \\
\text { - joint tenderness (score of } 0.5 \text { vs } 0.8 ; B L \approx 1.5 \text { ); } \boldsymbol{p}=\mathbf{0} .006 \text { vs } \text { DIC }^{\mathbf{b}}\end{array}$ \\
\hline $\begin{array}{l}\text { Pareek et al. } 2013^{28}(r, d b \text {, } \\
\mathrm{mc})\end{array}$ & $\begin{array}{l}\text { ACE vs DIC }(6 \\
\text { weeks })\end{array}$ & $\begin{array}{l}\text { Both groups had improved WOMAC total scores (mean change }-19 \text { vs }-19 \text {; BL } 43 \text { ) Overall efficacy assessed as } \\
\text { good or excellent by patients ( } 42 \% \text { vs } 40 \% \text { ) and physicians ( } 42 \% \text { vs } 40 \% \text { ) }\end{array}$ \\
\hline \multicolumn{3}{|l|}{ Vs nabumetone } \\
\hline $\begin{array}{l}\text { Gijón-Baños et al. } 1997^{54} \\
(\mathrm{r}, \text { ol, mc) }\end{array}$ & $\begin{array}{l}\text { ACE vs NAB (12 } \\
\text { weeks) }\end{array}$ & Both groups had improved GSI scores; both p significant vs BL \\
\hline Paul et al. $2009^{22}(r, \mathrm{db}, \mathrm{sc})$ & $\begin{array}{l}\text { ACE vs } N A B \text { vs } P L \\
\text { (4 weeks) }\end{array}$ & Disease status assessed as very/moderately well in $38 \%$ vs $47 \%$ vs $8 \%$ of patients \\
\hline \multicolumn{3}{|l|}{ Vs naproxen } \\
\hline $\begin{array}{l}\text { Kornasoff et al. } 1997^{24}(r, \\
\text { db, mc) }\end{array}$ & $\begin{array}{l}\text { ACE vs NAP (I2 } \\
\text { weeks) }\end{array}$ & $\begin{array}{l}\text { Both groups had improved: } \\
\text { - Functional capacity ( } 81 \% \text { vs } 84 \% \text { of patients) } \\
\text { - Joint swelling ( } 73 \% \text { vs } 75 \% \text { of patients) } \\
\text { - Joint erythema ( } 43 \% \text { vs } 48 \% \text { of patients) Joint tenderness on pressure ( } 82 \% \text { vs } 86 \% \text { of patients) } \\
\text { - Patient's overall assessment ( } 72 \% \text { vs } 72 \% \text { of patients) }\end{array}$ \\
\hline \multicolumn{3}{|l|}{ Vs piroxicam } \\
\hline Torri et al. $1994^{20}(r, d b)$ & $\begin{array}{l}\text { ACE vs PIR (12 } \\
\text { weeks) }\end{array}$ & $\begin{array}{l}\text { Both groups had improved: } \\
\text { - GSI score }(7.3 \text { vs } 7.2 ; B L \approx 12) \text {; both } p<0.01 \text { vs } B L \\
\text { - knee flexion }\left(106^{\circ} \text { vs } 106^{\circ} ; B L \approx 97^{\circ}\right) \text {; both } p<0.01 \text { vs } B L \\
\text { - knee extension }\left(1^{\circ} \text { vs } 0.8^{\circ} ; B L \approx 3^{\circ}\right) \text {; both } p<0.01 \text { vs } B L\end{array}$ \\
\hline $\begin{array}{l}\text { Pérez-Busquier et al. } \\
1997^{19}(\mathrm{r}, \mathrm{db}, \mathrm{mc})\end{array}$ & $\begin{array}{l}\text { ACE vs PIR (8 } \\
\text { weeks) }\end{array}$ & $\begin{array}{l}\text { Both groups had improved: } \\
\text { - OSI score }(7.5 \text { vs } 7.2 ; \mathrm{BL} \approx 12) \text {; both } p<0.00 \mathrm{I} \text { vs } \mathrm{BL} \\
\text { - knee function score }(4 \text { vs } 4 ; \mathrm{BL} \approx 7) \text {; both } p<0.001 \text { vs } \mathrm{BL} \\
\text { - knee flexion }\left(129^{\circ} \text { vs } 126^{\circ} \mathrm{BL} \approx 117^{\circ}\right) \text {; both } p<0.001 \text { vs } \mathrm{BL} \\
\text { - knee extension }\left(2^{\circ} \text { vs } 3^{\circ} ; \mathrm{BL} \approx 4.5^{\circ}\right) \text {; both } p<0.005 \text { vs } \mathrm{BL}\end{array}$ \\
\hline \multicolumn{3}{|l|}{ Vs paracetamol } \\
\hline $\begin{array}{l}\text { Batlle-Gualda et al. } 2007^{21} \\
(\mathrm{r}, \mathrm{db}, \mathrm{mc})\end{array}$ & $\begin{array}{l}\text { ACE vs PAR (6 } \\
\text { weeks) }\end{array}$ & $\begin{array}{l}\text { Both groups had improved: } \\
\text { - OSI score }{ }^{\text {a }} \text { (change }-2.4 \text { vs. }-0.97 ; B L \approx 11 \text { ); both } p<0.01 \text { vs } B L \text { and } p=0.004 \text { vs } \mathbf{P A R}^{\mathbf{b}} \\
\text { - PGA score }{ }^{\text {a }} \text { (change }-0.7 \text { vs }-0.3 ; B L \approx 2.5 \text { ); both } p<0.001 \text { vs } B L \text { and } p=0.017{\text { vs } \mathbf{P A R}^{\mathbf{b}}} \\
\text { - PhGA score }{ }^{\text {a }} \text { (change }-0.6 \text { vs }-0.4 ; B L \approx 3.2 \text { ); both } p<0.001 \text { vs } B L p=0.041 \text { vs } \mathbf{P A R}^{\mathbf{b}} \\
\text { - WOMAC total score (mean treatment difference } 4.28 \text { ); } \boldsymbol{p}=\mathbf{0 . 0 1 8} \text { vs } \mathbf{P A R}^{\mathbf{b}}\end{array}$ \\
\hline
\end{tabular}

Notes: aPrimary endpoint. ' Statistically significant differences between treatment groups are bolded.

Abbreviations: ACE, aceclofenac $100 \mathrm{mg}$ twice daily; BL, baseline; db, double-blind; DIC, diclofenac $50 \mathrm{mg}$ three times daily or $75 \mathrm{mg}$ twice daily; GSI, Gonarthrosis Severity Index; mc, multicenter; NAB, nabumetone I-2 g daily or 750 mg twice daily; NS, nonsignificant; OA, osteoarthritis; ol, open-label; OSI, Lequesne Osteoarthritis Severity Index; PAR, paracetamol I g three times daily; PGA, patient's global assessment; PhGA, physician's global assessment; PID, pain intensity difference; PIR, piroxicam $20 \mathrm{mg}$ once daily; r, randomized; sb, single-blind; sc, single center; WOMAC, Western Ontario and McMaster University. 
Table 3 Efficacy of Aceclofenac in Patients with Ankylosing Spondylitis

\begin{tabular}{|c|c|c|c|}
\hline \multirow[t]{2}{*}{ Study (design) } & \multirow{2}{*}{$\begin{array}{l}\text { Treatment } \\
\text { (duration) }\end{array}$} & \multirow{2}{*}{$\begin{array}{l}\text { No. of } \\
\text { eval. pts }\end{array}$} & \multirow{2}{*}{$\begin{array}{l}\text { Outcomes (ACE vs Comparator) } \\
\text { (Mean Values Reported) }\end{array}$} \\
\hline & & & \\
\hline Versus indomethacin & & & Changes from $B L$ \\
\hline $\begin{array}{l}\text { Batlle-Gualda et al } \\
1996^{30}(\mathrm{r}, \mathrm{db}, \mathrm{mc})\end{array}$ & $\begin{array}{l}\text { ACE vs IND } \\
\text { (12 weeks) }\end{array}$ & $\begin{array}{l}155 \text { vs } \\
153\end{array}$ & 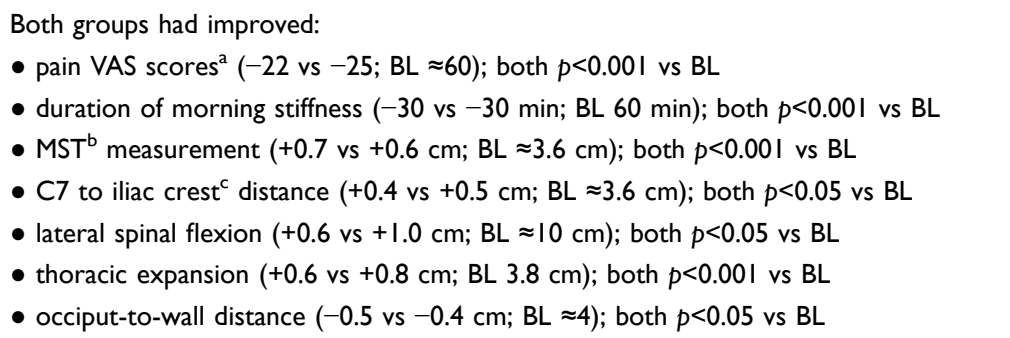 \\
\hline Vs naproxen & & & Mean values \\
\hline $\begin{array}{l}\text { Pasero et al. } 1994^{31} \text { ( } r \\
\mathrm{db}, \mathrm{mc})\end{array}$ & $\begin{array}{l}\text { ACE vs NAP } \\
\text { (12 weeks) }\end{array}$ & 60 vs 66 & $\begin{array}{l}\text { Both groups had improved: } \\
\left.\text { - pain VAS scores }{ }^{\mathrm{a}} \text { ( } 25 \text { vs } 29 ; \mathrm{BL} \approx 53\right) \text {; both } p<0.0 \mathrm{I} \text { vs } \mathrm{BL} \\
\text { - } \text { MST }^{\mathrm{b}} \text { measurement }(14 \mathrm{vs} 14 \mathrm{~cm} ; \mathrm{BL} \approx 13 \mathrm{~cm} \text { ); both } p<0.0 \text { I vs } \mathrm{BL} \\
\text { - hand-to-floor distance }(17 \mathrm{vs} 20 \mathrm{~cm} ; \mathrm{BL} 22 \text { vs } 26 \mathrm{~cm} \text { ); both } p<0.0 \text { I vs } \mathrm{BL} ; \boldsymbol{p}<0.05 \text { vs } \\
\text { NAP }\end{array}$ \\
\hline Vs tenoxicam & & & Changes from $B L$ \\
\hline $\begin{array}{l}\text { Villa Alcázar et al } \\
1996^{32} \text { (r, db, mc) }\end{array}$ & $\begin{array}{l}\text { ACE vs TEN } \\
\text { (1 } 2 \text { weeks) }\end{array}$ & $\begin{array}{l}135 \text { vs } \\
138\end{array}$ & 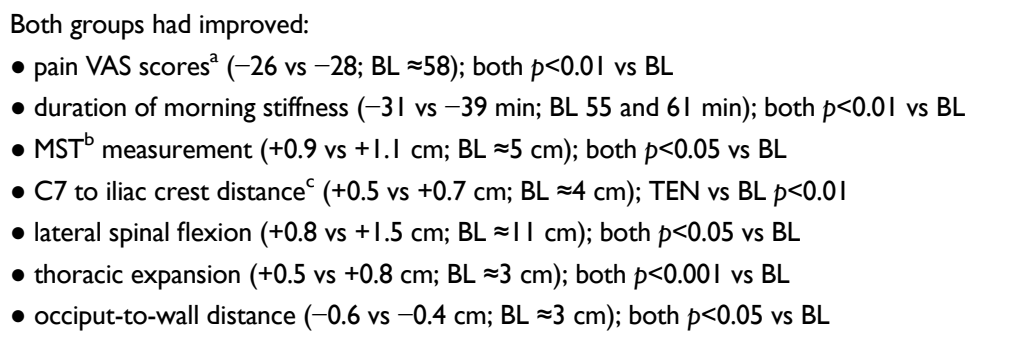 \\
\hline
\end{tabular}

Notes: a VAS score assessed on a 0-100 mm. 'Increase in length of the $10 \mathrm{~cm}$ of spine above L5 after extension (flexion) of the lumbar spine. ${ }^{\mathrm{C}} \mathrm{Overall}$ spinal movement as shown by $C 7$ to iliac crest line measurement. 'Statistically significant difference between treatment groups is bolded.

Abbreviations: ACE, aceclofenac 100 mg twice daily; BL, baseline; IND, indomethacin $100 \mathrm{mg}$ daily; MST, Modified Schöber's test; NS, nonsignificant; NAP, naproxen $500 \mathrm{mg}$ twice daily; TEN, tenoxicam $20 \mathrm{mg}$ once daily; VAS, visual analogue scale.

with $31 \%$ of patients receiving aceclofenac alone achieved the Assessment of SpondyloArthritis International Society (ASAS) 20 response criteria $(p=0.047)$. No significant differences were seen between combination therapy and monotherapy in the reduction from baseline in the pain visual analog scale (VAS) score (46\% vs $26 \%$ ) or the Bath Ankylosing Spondylitis Disease Activity Index score (mean change -2.2 vs -1.5 ; baseline $\approx 5.5$ ), as well as improvements in measures of spinal mobility or health-related quality of life. The small sample size is a major limitation of this study, which may account for marginal differences between the treatment groups. ${ }^{33}$

\section{Rheumatoid Arthritis}

Aceclofenac $100 \mathrm{mg}$ twice daily was at least as effective as diclofenac, indomethacin, ketoprofen, and tenoxicam in reducing pain intensity and joint inflammation, and improving morning stiffness and hand grip strength in four 12- to 24-week, randomized clinical trials in patients with confirmed active RA (see Table 4 for dosage details). Following $\leq 24$ weeks' therapy, joint inflammation (assessed by Ritchie Index scores) was significantly $(p<0.01)$ reduced from baseline with aceclofenac and comparators (diclofenac, ketoprofen, and tenoxicam), with no significant betweengroup differences (Table 4). ${ }^{34-36}$ Significant improvements in Ritchie Index scores were seen from day 15 onwards in patients receiving aceclofenac, ${ }^{34-36}$ diclofenac, ${ }^{35}$ and tenoxicam $^{36}$ (all $p<0.01$ vs baseline), and from day 30 onwards in patients receiving ketoprofen (all $p<0.05$ vs baseline). ${ }^{35}$ The numbers of painful and swollen joints were significantly reduced with both aceclofenac and indomethacin (Table 4). ${ }^{37}$ 
Table 4 Efficacy of Aceclofenac in Patients with Rheumatoid Arthritis

\begin{tabular}{|c|c|c|c|}
\hline \multirow[t]{2}{*}{ Study (Design) } & \multirow{2}{*}{$\begin{array}{l}\text { Treatment } \\
\text { (Duration) }\end{array}$} & \multirow{2}{*}{$\begin{array}{l}\text { No. of } \\
\text { Eval. } \\
\text { pts }\end{array}$} & \multirow[t]{2}{*}{ Outcomes (ACE vs Comparator) } \\
\hline & & & \\
\hline \multicolumn{4}{|l|}{ Versus diclofenac } \\
\hline $\begin{array}{l}\text { Pasero et al } \\
1995^{35}(\mathrm{r}, \mathrm{db}, \mathrm{mc})\end{array}$ & $\begin{array}{l}\text { ACE vs DIC } \\
\text { ( } 24 \text { weeks) }\end{array}$ & $\begin{array}{l}131 \text { vs } \\
130\end{array}$ & $\begin{array}{l}\text { Both groups had improved: } \\
\text { - pain VAS scores }{ }^{\mathrm{a}} \text { (mean } 38 \text { vs } 37 ; \mathrm{BL} 62 \text { and } 58 \text { ); both } p<0.0 \mathrm{I} \text { vs } \mathrm{BL} \\
\text { - duration of morning stiffness (mean } 49 \text { vs } 46 \mathrm{~min} ; \mathrm{BL} 8 \mathrm{I} \text { and } 85 \mathrm{~min} \text { ); both } p<0.0 \mathrm{I} \text { vs } \mathrm{BL} \\
\text { - Ritchie index (mean I2 vs I2; } \mathrm{BL} 22 \text { vs } 20 \text { ); both } p<0.0 \mathrm{I} \text { vs } \mathrm{BL} ; \boldsymbol{p}<0.05 \text { vs } \mathrm{DIC}^{\mathrm{a}} \\
\text { - hand grip (mean } 9 \mathrm{I} \text { vs } 95 \mathrm{mmHg} ; \mathrm{BL} 75 \text { and } 8 \mathrm{ImHg} \text { ); both } p<0.0 \mathrm{I} \text { vs } \mathrm{BL}\end{array}$ \\
\hline \multicolumn{4}{|l|}{ Vs indomethacin } \\
\hline $\begin{array}{l}\text { Kornasoff et al } \\
1996^{37}(\mathrm{r}, \mathrm{db}, \mathrm{mc})\end{array}$ & $\begin{array}{l}\text { ACE vs IND } \\
\text { (12 weeks) }\end{array}$ & $\begin{array}{l}109 \text { vs } \\
110\end{array}$ & $\begin{array}{l}\text { Both groups had improved: } \\
\left.\text { - pain VAS scores }{ }^{\mathrm{a}} \text { at rest as per patient's assessment ( }-23 \text { vs }-19\right) \\
\text { - pain VAS scores at rest as per physician's assessment ( } 65 \% \text { vs } 67 \% \text { of patients) } \\
\text { - duration of morning stiffness (median }-\mathrm{I} \text { vs }-0.7 \mathrm{~h} ; \mathrm{BL} 2 \mathrm{~h} \text { ); both } p<0.05 \\
\text { - number of painful (median change }-9 \mathrm{vs}-6 \text {; BL } 24 \text { ) and swollen ( }-6 \text { vs }-6 \text {; BL I7) joints; } \\
\text { all } p<0.05 \text { vs BL } \\
\text { - grip strength right and left hand (median } 8 \text { vs } 10 \mathrm{mmHg} \text { for both); all } p<0.05 \text { vs BL }\end{array}$ \\
\hline \multicolumn{4}{|l|}{ Vs ketoprofen } \\
\hline $\begin{array}{l}\text { Martín-Mola et al } \\
1995^{34}(\mathrm{r}, \mathrm{db}, \mathrm{mc})\end{array}$ & $\begin{array}{l}\text { ACE vs KET } \\
\text { (I } 2 \text { weeks })\end{array}$ & 87 vs 82 & 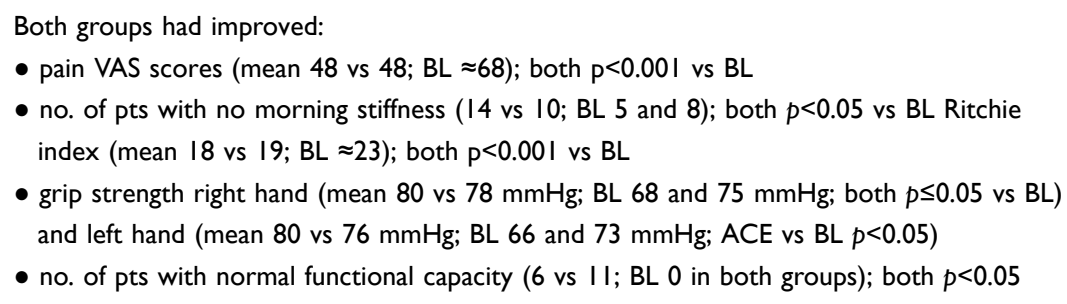 \\
\hline \multicolumn{4}{|l|}{ Vs tenoxicam } \\
\hline $\begin{array}{l}\text { Pérez-Ruiz et al } \\
1996^{36}(\mathrm{r}, \mathrm{db}, \mathrm{mc})\end{array}$ & $\begin{array}{l}\text { ACE vs TEN } \\
\text { (1 } 2 \text { weeks) }\end{array}$ & $\begin{array}{l}145 \text { vs } \\
147\end{array}$ & $\begin{array}{l}\text { Both groups had improved: } \\
\text { - pain VAS scores (mean } 42 \text { vs } 39 ; B L \approx 62 \text { ); both } p<0.01 \text { vs } B L \text { no. of pts with } \\
\text { - no morning stiffness ( } 24 \text { vs } 24 ; B L 0 \text { and I); both } p<0.00 \text { I vs } B L \\
\text { - Ritchie index (mean } 14 \text { vs I2; } B L \approx 22 \text { ); both } p<0.01 \text { vs } B L \\
\text { - grip strength right hand (mean } 94 \text { vs } 99 \mathrm{mmHg} B \mathrm{BL} \approx 80 \mathrm{mmHg} \text {; both } p<0.01 \text { vs } B L \text { ) and } \\
\text { left hand (mean } 92 \text { vs } 94 \mathrm{mmHg}, \mathrm{BL} \approx 81 \mathrm{mmHg} \text { both } p<0.05 \text { ) }\end{array}$ \\
\hline
\end{tabular}

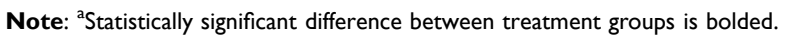

Abbreviations: ACE, aceclofenac 100 mg twice daily; DIC, 50 mg three times daily; IND, indomethacin 100 mg daily; MST, Modified Schöber's test; NS, nonsignificant; NAP, naproxen $500 \mathrm{mg}$ twice daily; TEN, tenoxicam $20 \mathrm{mg}$ once daily; VAS, visual analogue.

Pain VAS scores were significantly reduced with aceclofenac in three of four studies, ${ }^{34-36}$ with significant improvements also seen with diclofenac, ketoprofen, and tenoxicam (but not with indomethacin) (Table 4). ${ }^{3-37}$ No significant differences between treatment groups were seen in any of the studies. Where assessed, a significant improvement in pain intensity was observed from day 15 onwards in aceclofenac, diclofenac, and tenoxicam recipients. ${ }^{35,36}$

The duration of morning stiffness was reduced, ${ }^{35,37}$ the number of patients with no morning stiffness was increased, ${ }^{34,36}$ and hand grip strength was increased ${ }^{34-37}$ to a similar extent with aceclofenac and comparators (Table 4). Where assessed, significant improvements in morning stiffness were seen by day 15 with aceclofenac, diclofenac, and tenoxicam (all $p<0.01$ vs baseline); significant improvements in hand grip strength were seen by day $15^{36}$ or day $30^{35}$ with aceclofenac, day 15 with tenoxicam ${ }^{36}$ and day 30 with diclofenac. ${ }^{35}$

\section{Low Back Pain}

Aceclofenac $100 \mathrm{mg}$ twice daily has also demonstrated efficacy in patients with LBP. A 10-day randomized, 
double-blind, multicenter non-inferiority study in patients with acute LBP (uncomplicated acute lumbosacral pain) suffering from degenerative spinal disorders showed that aceclofenac $100 \mathrm{mg}$ twice daily $(\mathrm{n}=100)$ was non-inferior (primary endpoint) and superior to diclofenac $75 \mathrm{mg}$ twice daily $(n=105)$ in terms of analgesic efficacy (mean change in pain VAS score 61.6 vs $57.3 \mathrm{~mm}$; baseline $\approx 79 \mathrm{~mm}$ ); however, the between-group difference in the score was not considered clinically relevant. ${ }^{38}$

\section{Safety and Tolerability Profile}

Aceclofenac was generally well tolerated, with a safety profile consistent with that expected of NSAIDs. ${ }^{1,2}$ In clinical studies and during post-marketing experience, the most common adverse events (AEs) with aceclofenac were gastrointestinal (GI) disorders (dyspepsia, abdominal pain, nausea, and diarrhea), dizziness and increased hepatic enzymes. ${ }^{3}$ There have also been rare $(\geq 1 / 10,000$ to $<1 /$ 1000) occurrences of peptic ulcers or GI bleeding, and very rare $(<1 / 10,000)$ intestinal perforation, which may be fatal at times, particularly in the elderly. ${ }^{3}$

Aceclofenac appeared to be better tolerated than several other NSAIDs, including naproxen, piroxicam, indomethacin, and ketoprofen ${ }^{20,24,31,34,37}$ (Figure 1), and had a tolerability profile generally similar to that of tenoxicam $^{32,36}$ and paracetamol. $^{21}$ Furthermore, in an analysis of all spontaneous adverse reactions recorded in the pharmacovigilance database of the World Health Organization Collaborating Center for International Drug Monitoring during the first year after introduction of the drugs in the UK, the incidence rate (adverse reactions $/ 10^{6}$ defined daily dose) of total adverse reactions with aceclofenac (8.7; 95\% CI 6.1-12.0) was lower than with meloxicam (24.8; 95\% CI 23.1-26.6) or rofecoxib (52.6; 95\% CI 49.9-55.4). ${ }^{39}$ In terms of the seven groups of conditions most commonly associated with NSAIDs, aceclofenac had lower incidences of GI bleeding, abdominal pain and arterial hypertension than meloxicam or rofecoxib, and a lower incidence of liver toxicity, thromboembolic $\mathrm{CV}$ events and edema than rofecoxib. ${ }^{39}$

Aceclofenac has a lower incidence of GI AEs compared with other NSAIDs, which may result in lower withdrawal rates and increased treatment compliance. ${ }^{1,40}$ A large $(\mathrm{n}=$ 10,142 patients), 12-month prospective, observational study complying with Safety Assessment of Marketed Medicines (SAMM) guidelines showed that in patients with RA, OA, or AS receiving aceclofenac $(n=7890)$ or diclofenac $(n=2252)$, the overall incidence of AEs was
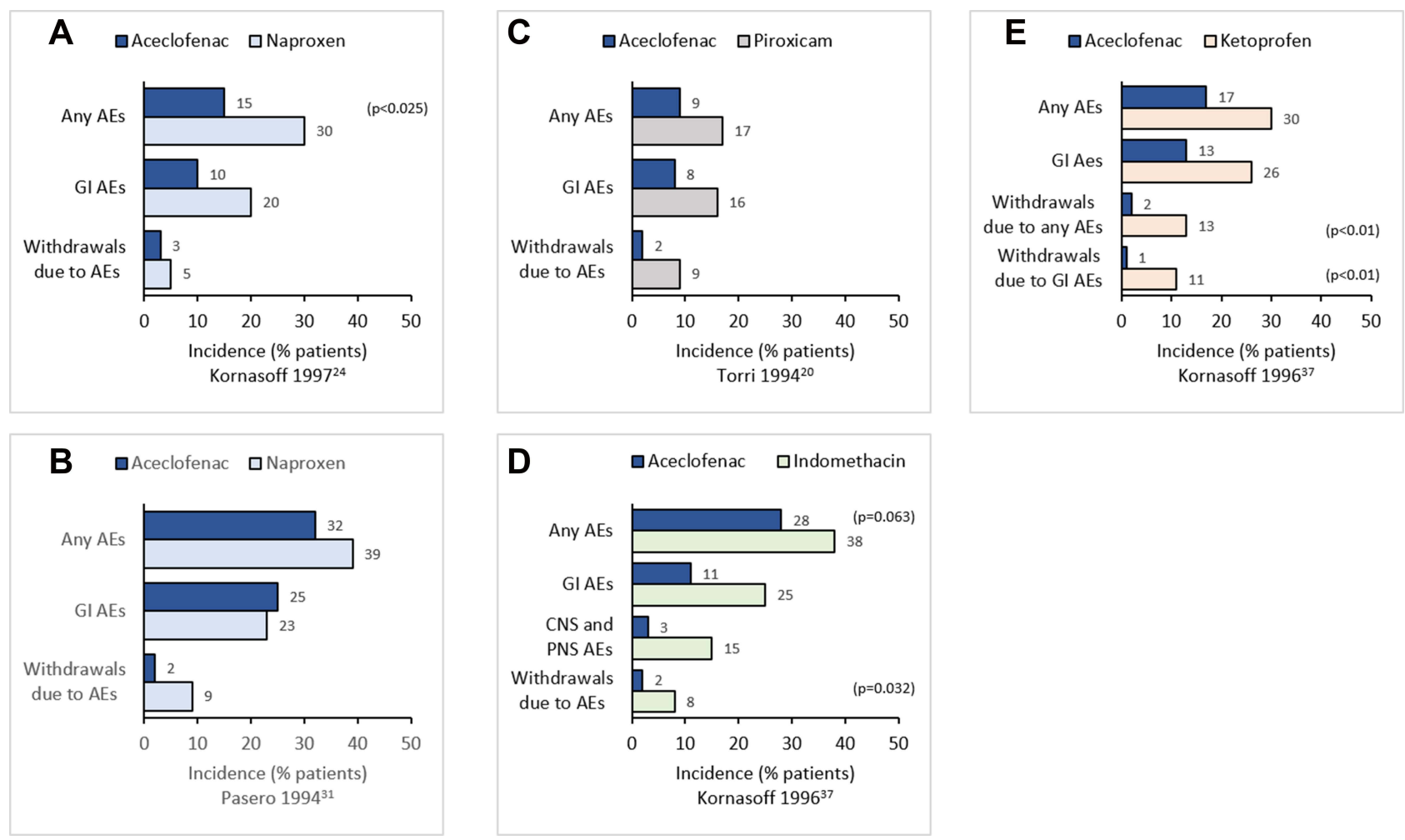

Figure I Tolerability of aceclofenac versus naproxen (A and B), piroxicam (C), indomethacin (D) and ketoprofen (E) in clinical studies. $p$ values reported where available. 
significantly lower in aceclofenac than diclofenac recipients (22 vs $27 \% ; p<0.001) .{ }^{41}$ Both NSAIDs were generally well tolerated, with most AEs of mild or moderate severity. The most common AEs in both treatment groups were mild or moderate GI AEs, which occurred in significantly fewer aceclofenac than diclofenac recipients $(11 \%$ vs $15 \%$; $p<0.001)$. GI AEs included dyspepsia (5.4\% vs $6.7 \%$; $p=0.017)$, abdominal pain $(2.5 \%$ vs $4.4 \% ; p<0.001)$, diarrhea $(1.5 \%$ vs $3.6 \%$; $p<0.001$ ), and nausea ( 1.6 vs $2.4 \%$; $p=0.01)$. Central Nervous System (CNS) disorders, mostly mild or moderate, occurred in $<3 \%$ of patients in the two treatment groups, with a higher incidence of aceclofenac than diclofenac recipients ( $3 \%$ vs $2 \%$; $p=0.007)$, largely because of higher incidences of dizziness, depression, and headache with aceclofenac. ${ }^{41}$

In a 6-week randomized controlled trial in patients with OA, significantly fewer patients receiving aceclofenac $(\mathrm{n}=$ $284)$ than diclofenac $(n=285)$ had GI AEs (primary outcome; $57 \%$ vs $74 \%$; $p<0.001$ ), all of which were of mild or moderate severity. ${ }^{28}$ The most common (incidence $>5 \%$ ) GI AEs in aceclofenac and diclofenac recipients were dyspepsia ( $28 \%$ vs $38 \%$; $p=0.014$ ), abdominal pain $(19 \%$ vs $26 \% ; p=0.037)$, and nausea ( 7 vs $6 \%$ ). Two patients in each treatment group withdrew from the study because of GI AEs. ${ }^{28}$

Two meta-analyses provide further evidence for the better GI tolerability of aceclofenac relative to other NSAIDs. A meta-analysis of seven randomized clinical trials of aceclofenac in OA found no significant difference between aceclofenac and comparators (diclofenac, naproxen, piroxicam, and paracetamol) in terms of the occurrence of AEs (relative risk [RR] 0.90; 95\% CI 0.72, 1.12 ; $\mathrm{I}^{2} 58 \%$ ), withdrawal rates (RR $0.84 ; 95 \%$ CI 0.67 , $1.05 ; \mathrm{I}^{2}=9 \%$ ), AE-related withdrawal rates (RR 0.76; $95 \%$ CI $0.51,1.14$ ) or withdrawal rates due to GI AEs (RR $1.30 ; 95 \%$ CI $0.62,2.74) .{ }^{29}$ However, the relative risk of

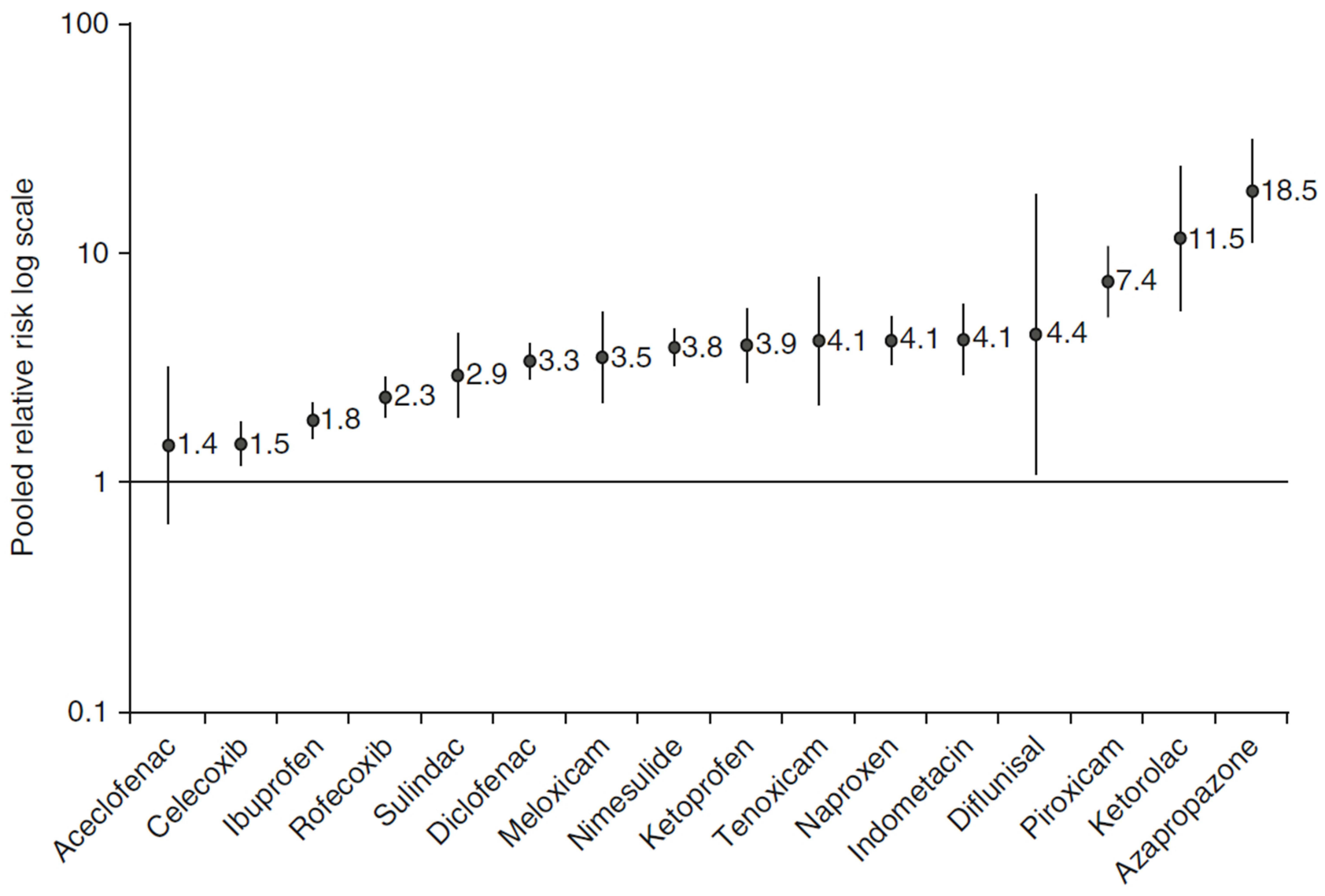

NSAIDs

Figure 2 The pooled relative risk (RR) of upper gastrointestinal complications with the use of NSAIDs versus non-use of NSAIDs according to a systematic review and meta-analysis of observational studies. Vertical bars represent $95 \%$ confidence intervals. Of note, rofecoxib has been withdrawn from the market due to cardiovascular adverse events. Republished from Springer Nature, Castellsague J, Riera-Guardia N, Calingaert B, et al. Individual NSAIDs and upper gastrointestinal complications: a systematic review and meta-analysis of observational studies (the SOS project). Drug Saf. 20I2;35(I2):II27-II46; permission conveyed through Copyright Clearance Center, Inc. ${ }^{42}$ 
GI AEs (reported in four trials) was 31\% lower with aceclofenac than the comparators, diclofenac and piroxicam (RR 0.69; 95\% CI 0.57, 0.83; $p<0.0001 ; \mathrm{I}^{2}=12 \%$ ). ${ }^{29}$

A meta-analysis of 28 observational studies calculated the RR of upper GI complications with the use of NSAIDs versus non-use of NSAIDs and found that aceclofenac, ibuprofen, and celecoxib had RRs of $<2$, while other NSAIDs had RRs of 2 to $>5$ (Figure 2). ${ }^{42}$ It should be noted that there was no significant increase in the risk of upper GI complications with the use of aceclofenac versus non-use of NSAIDs.

NSAIDs, like aceclofenac, have also been associated with renal, CV and dermatological AEs. ${ }^{43,44}$ However, in clinical studies and during post-marketing experience with aceclofenac, there were uncommon to very rare occurrences of cardiac disorders (cardiac failure -rare- and palpitations -very rare- occurrences), renal disorders (eg increased blood urea and blood creatinine uncommon occurrences), and skin and subcutaneous tissue disorders (eg pruritus, rash and dermatitis uncommon occurrences) (uncommon $\geq 1 / 1000$ to $<1 / 100$; rare $\geq 1 / 10,000$ to $<1 / 1000$; very rare $<1 / 10,000) .{ }^{3}$

Concerning special populations, such as elderly patients, the pharmacokinetics of aceclofenac are not significantly altered in the elderly and dosage modifications are not required. ${ }^{3}$ However, the elderly are more likely to be suffering from impaired renal, CV or hepatic function, at higher risk of GI bleeding, ulceration or perforation, and receiving concomitant medication, which may increase the risk of interactions and adverse reactions. ${ }^{3,44} 46$ The risk of GI bleeding or ulceration may be increased when NSAIDs are used concomitantly with systemic corticosteroids, anticoagulants (eg warfarin), selective serotonin-reuptake inhibitors or antiplatelet agents such as aspirin. Coadministration of NSAIDs and methotrexate may increase plasma levels of methotrexate, resulting in increased toxicity. $3,44,47$

Given the higher risk of adverse reactions with NSAIDs in the elderly, particularly GI bleeding, it is recommended the lowest effective dose of NSAID be used and for the shortest possible duration.3 Elderly patients should be monitored regularly for GI bleeding and renal insufficiency during NSAID therapy ${ }^{3}$

\section{Discussion}

Pharmacological treatment is the mainstay for pain management in OA, inflammatory arthritis (including RA and AS) and LBP, with treatment options including NSAIDs and analgesics (eg paracetamol, codeine, and other opiatelike drugs). ${ }^{48,49}$ Oral NSAIDs are the initial treatment of choice for patients with knee, hip, and/or hand OA, and are recommended over all other available oral medications. ${ }^{50}$ NSAIDs are also recommended as firstline therapy for patients with AS suffering from pain and stiffness, with continuous use preferred in patients who are symptomatic and respond well to treatment. ${ }^{51,52}$ Likewise, NSAIDs are first-line treatment for $\mathrm{LBP}^{49}$ and are also an option for the management of pain in RA. ${ }^{48}$

The NSAID aceclofenac is a potent COX-2 inhibitor with a well-established efficacy and tolerability profile in patients with musculoskeletal disorders. Several randomized clinical studies in patients with OA, AS, RA, or LBP, demonstrated that aceclofenac was at least as effective as diclofenac, nabumetone, naproxen, piroxicam, indomethacin, tenoxicam, and ketoprofen in terms of reducing pain and/or improving functional capacity. Aceclofenac is generally well tolerated, with the most common AEs being GI disorders, dizziness, and increased hepatic enzymes.

Notably, evidence indicates that aceclofenac has better GI tolerability than that of other NSAIDs. Aceclofenac had a significantly lower incidence of GI AEs in two clinical studies $^{28,41}$ and lower incidences of GI bleeding and abdominal pain in a pharmacovigilance data analysis. ${ }^{39}$ These findings were supported by two meta-analyses, with one analysis showing that aceclofenac had a $31 \%$ lower risk of GI AEs than diclofenac or piroxicam. ${ }^{29}$ In the second meta-analysis, among the NSAIDs assessed, aceclofenac had the lowest (and nonsignificant) risk of upper GI complications. $^{42}$

Given the general similarity in efficacy of NSAIDs, the costs associated with NSAID therapy are likely to be largely a function of drug acquisition costs and the comparative incidence of adverse events associated with the drugs. A pharmacoeconomic analysis conducted using 1996 cost data estimated that the iatrogenic cost factor (ICF) of aceclofenac was significantly lower than that of other NSAIDs (diclofenac, indomethacin, ketoprofen, naproxen, and tenoxicam) used in the treatment of RA, OA and AS. ${ }^{53}$ The study found no significant difference between the ICF of aceclofenac and piroxicam. ${ }^{53}$ Additional well-designed pharmacoeconomic analyses based on current costs associated with NSAID therapy are needed to confirm the potential costeffectiveness of aceclofenac in these indications.

To conclude, aceclofenac is generally well tolerated and effective in reducing pain and/or improving functional capacity in chronic (OA, AS, RA) and acute (eg LBP) painful musculoskeletal conditions. It is at least as effective as other NSAIDs and appears to have a more favorable GI profile. Thus, current evidence indicates that aceclofenac is a useful 
option for the management of pain and inflammation across a wide range of painful conditions.

\section{Acknowledgments}

Medical writing support, under the guidance of the authors, was provided by Content Ed Net, with funding from Almirall.

\section{Funding}

Writing assistance was funded by Almirall.

\section{Disclosure}

The authors report no conflicts of interest in relation to this article.

\section{References}

1. Dooley M, Spencer CM, Dunn CJ. Aceclofenac: a reappraisal of its use in the management of pain and rheumatic disease. Drugs. 2001;61(9):1351-1378. doi:10.2165/00003495-200161090-00012

2. Brogden RN, Wiseman LR. Aceclofenac. A review of its pharmacodynamic properties and therapeutic potential in the treatment of rheumatic disorders and in pain management. Drugs. 1996;52 (1):113-124. doi:10.2165/00003495-199652010-00008

3. Almirall Ltd. PRESERVEX ${ }^{\circledR}$ (aceclofenac) $100 \mathrm{mg}$ film-coated tablets: UK summary of prescribing characteristics; 2018. Available from: https:// www.medicines.org.uk/emc/product/6578/smpc. Accessed November 6, 2021

4. Almirall SA. Airtal (aceclofenac) $100 \mathrm{mg}$ film-coated tablets: summary of product characteristics; 2014. Available from: https://cima.aemps.es/ cima/dochtml/ft/59024/FT_59024.html. Accessed November 6, 2021.

5. Ricciotti E, FitzGerald GA. Prostaglandins and inflammation. Arterioscler Thromb Vasc Biol. 2011;31(5):986-1000. doi:10.1161/ ATVBAHA.110.207449

6. Blackwell KA, Raisz LG, Pilbeam CC. Prostaglandins in bone: bad cop, good cop? Trends Endocrinol Metab. 2010;21(5):294-301. doi:10.1016/j.tem.2009.12.004

7. Atchison JW, Herndon CM, Rusie E. NSAIDs for musculoskeletal pain management: current perspectives and novel strategies to improve safety. J Manag Care Pharm. 2013;19(9Suppl A):S3-19.

8. Alvarez-Soria MA, Largo R, Santillana J, et al. Long term NSAID treatment inhibits COX-2 synthesis in the knee synovial membrane of patients with osteoarthritis: differential proinflammatory cytokine profile between celecoxib and aceclofenac. Ann Rheum Dis. 2006;65(8):998-1005. doi:10.1136/ard.2005.046920

9. Alvarez-Soria MA, Herrero-Beaumont G, Moreno-Rubio J, et al. Long-term NSAID treatment directly decreases COX-2 and mPGES-1 production in the articular cartilage of patients with osteoarthritis. Osteoarthritis Cartilage. 2008;16(12):1484-1493. doi:10.1016/j.joca.2008.04.022

10. Lonappan L, Brar SK, Das RK, Verma M, Surampalli RY. Diclofenac and its transformation products: environmental occurrence and toxicity - A review. Environ Int. 2016;96:127-138. doi:10.1016/j. envint.2016.09.014

11. Sharma P. Aceclofenac as a potential threat to critically endangered vultures in India: a review. $J$ Raptor Res. 2012;46(3):314-318, 315 . doi:10.3356/JRR-11-66.1

12. World Health Organization. Chronic rheumatic conditions; 2020. Available from: https://www.who.int/chp/topics/rheumatic/en/. Accessed November 6, 2021.
13. Hoy D, Bain C, Williams G, et al. A systematic review of the global prevalence of low back pain. Arthritis Rheum. 2012;64 (6):2028-2037. doi:10.1002/art.34347

14. Cross M, Smith E, Hoy D, et al. The global burden of hip and knee osteoarthritis: estimates from the global burden of disease 2010 study. Ann Rheum Dis. 2014;73(7):1323-1330. doi:10.1136/annrheumdis2013-204763

15. Cross M, Smith E, Hoy D, et al. The global burden of rheumatoid arthritis: estimates from the global burden of disease 2010 study. Ann Rheum Dis. 2014;73(7):1316-1322. doi:10.1136/annrheumdis-2013204627

16. Stolwijk C, van Onna M, Boonen A, van Tubergen A. Global prevalence of spondyloarthritis: a systematic review and meta-regression analysis. Arthritis Care Res. 2016;68(9):1320-1331. doi:10.1002/ acr.22831

17. Global Burden of Disease Study 2013 Collaborators. Global, regional, and national incidence, prevalence, and years lived with disability for 301 acute and chronic diseases and injuries in 188 countries, 1990-2013: a systematic analysis for the Global Burden of Disease Study 2013. Lancet. 2015;386 (9995):743-800.

18. Dalys GBD, Collaborators H. Global, regional, and national disability-adjusted life-years (DALYs) for 333 diseases and injuries and healthy life expectancy (HALE) for 195 countries and territories, 1990-2016: a systematic analysis for the Global Burden of Disease Study 2016. Lancet. 2017;390(10100):1260-1344.

19. Pérez Busquier M, Calero E, Rodríguez M, et al. Comparison of aceclofenac with piroxicam in the treatment of osteoarthritis. Clin Rheumatol. 1997;16(2):154-159. doi:10.1007/BF02247844

20. Torri G, Vignati C, Agrifoglio E. Aceclofenac versus piroxicam in the management of osteoarthritis of the knee: a double-blind controlled study. Curr Ther Res Clin Exp. 1994;55(5):576-583. doi:10.1016/S0011-393X(05)80189-4

21. Batlle-Gualda E, Román Ivorra J, Martín-Mola E, et al. Aceclofenac vs paracetamol in the management of symptomatic osteoarthritis of the knee: a double-blind 6-week randomized controlled trial. Osteoarthritis Cartilage. 2007;15(8):900-908. doi:10.1016/j.joca. 2007.02.008

22. Paul S, Das N, Ghosh S. The effects of aceclofenac and nabumetone in osteoarthritis. JNMA J Nepal Med Assoc. 2009;48(174):121-125. doi:10.31729/jnma.226

23. Ward DE, Veys EM, Bowdler JM, Roma J. Comparison of aceclofenac with diclofenac in the treatment of osteoarthritis. Clin Rheumatol. 1995;14(6):656-662. doi:10.1007/BF02207932

24. Kornasoff D, Frerick H, Bowdler J, Montull E. Aceclofenac is a well-tolerated alternative to naproxen in the treatment of osteoarthritis. Clin Rheumatol. 1997;16(1):32-38. doi:10.1007/BF0 2238760

25. Pareek A, Chandanwale AS, Oak J, Jain UK, Kapoor S. Efficacy and safety of aceclofenac in the treatment of osteoarthritis: a randomized double-blind comparative clinical trial versus diclofenac - an Indian experience. Curr Med Res Opin. 2006;22(5):977-988. doi:10.1185/ 030079906X104722

26. Patil P, Jaida J, Palani A, Rao K. A comparative study of diclofenac and aceclofenac in the treatment of osteoarthritis patients. J Drug Delivery Ther. 2012;2(4). doi:10.22270/jddt. v22272i22274.22206

27. Sehgal A, Sehgal V, Singh R. Comparison of efficacy and tolerability of aceclofenac and diclofenac in osteoarthritis of knee joint. Int J Med Dental Sci. 2015;4:745. doi:10.19056/ijmdsjssmes/2015/ v4i2/79789

28. Pareek A, Chandurkar N. Comparison of gastrointestinal safety and tolerability of aceclofenac with diclofenac: a multicenter, randomized, double-blind study in patients with knee osteoarthritis. Curr Med Res Opin. 2013;29(7):849-859. doi:10.1185/03007995.2013.795139 
29. Patel PB, Patel TK. Efficacy and safety of aceclofenac in osteoarthritis: a meta-analysis of randomized controlled trials. Eur $J$ Rheumatol. 2017;4(1):11-18. doi:10.5152/eurjrheum.2017.160080

30. Batlle-Gualda E, Figueroa M, Ivorra J, Raber A. The efficacy and tolerability of aceclofenac in the treatment of patients with ankylosing spondylitis: a multicenter controlled clinical trial. Aceclofenac Indomethacin Study Group. J Rheumatol. 1996;23(7):1200-1206.

31. Pasero G, Ruju G, Marcolongo R. Aceclofenac versus naproxen in the treatment of ankylosing spondylitis: a double-blind, controlled study. Curr Ther Res. 1994;55:833-842. doi:10.1016/S0011-393X (05)80777-5

32. Villa Alcázar LF, de Buergo M, Rico Lenza H, Montull Fruitos E. Aceclofenac is as safe and effective as tenoxicam in the treatment of ankylosing spondylitis: a 3 month multicenter comparative trial. Spanish Study Group on Aceclofenac in Ankylosing Spondylitis. J Rheumatol. 1996;23(7):1194-1199.

33. Chang JK, Yu CT, Lee MY, et al. Tramadol/Acetaminophen combination as add-on therapy in the treatment of patients with ankylosing spondylitis. Clin Rheumatol. 2013;32(3):341-347. doi:10.1007/ s10067-012-2125-y

34. Martín-Mola E, Gijón-Baños J, Ansoleaga JJ. Aceclofenac in comparison to ketoprofen in the treatment of rheumatoid arthritis. Rheumatol Int. 1995;15(3):111-116. doi:10.1007/BF00302127

35. Pasero G, Marcolongo R, Serni U, Parnham MJ, Ferrer F. A multi-centre, double-blind comparative study of the efficacy and safety of aceclofenac and diclofenac in the treatment of rheumatoid arthritis. Curr Med Res Opin. 1995;13(6):305-315. doi:10.1185/ 03007999509110491

36. Pérez-Ruiz F, Alonso-Ruiz A, Ansoleaga JJ. Comparative study of the efficacy and safety of aceclofenac and tenoxicam in rheumatoid arthritis. Clin Rheumatol. 1996;15(5):473-477. doi:10.1007/BF02 229644

37. Kornasoff D, Maisenbacher J, Bowdler J, Raber A. The efficacy and tolerability of aceclofenac compared to indomethacin in patients with rheumatoid arthritis. Rheumatol Int. 1996;15(6):225-230. doi:10.10 07/BF00290375

38. Schattenkirchner M, Milachowski KA. A double-blind, multicentre, randomised clinical trial comparing the efficacy and tolerability of aceclofenac with diclofenac resinate in patients with acute low back pain. Clin Rheumatol. 2003;22(2):127-135. doi:10.1007/s10067-0030710-9

39. Raber A, Heras J, Costa J, Fortea J, Cobos A. Incidence of spontaneous notifications of adverse reactions with aceclofenac, meloxicam, and rofecoxib during the first year after marketing in the United Kingdom. Ther Clin Risk Manag. 2007;3(2):225-230. doi:10.2147/ tcrm.2007.3.2.225

40. Legrand E. Aceclofenac in the management of inflammatory pain. Expert Opin Pharmacother. 2004;5(6):1347-1357. doi:10.1517/1465 6566.5.6.1347

41. Huskisson EC, Irani M, Murray F. A large prospective open-label, multicentre SAMM study, comparing the safety of aceclofenac with diclofenac in patients with rheumatic disease. Eur $J$ Rheumatol Inflamm. 2000;17(1):1-7.

42. Castellsague J, Riera-Guardia N, Calingaert B, et al. Individual NSAIDs and upper gastrointestinal complications: a systematic review and meta-analysis of observational studies (the SOS project). Drug Saf. 2012;35(12):1127-1146. doi:10.1007/BF03261999
43. Scarpignato C, Lanas A, Blandizzi C, et al. Safe prescribing of nonsteroidal anti-inflammatory drugs in patients with osteoarthritis-an expert consensus addressing benefits as well as gastrointestinal and cardiovascular risks. BMC Med. 2015;13:55. doi:10.1186/s12916015-0285-8

44. Moore N, Pollack C, Butkerait P. Adverse drug reactions and drug-drug interactions with over-the-counter NSAIDs. Ther Clin Risk Manag. 2015;11:1061-1075.

45. Gooch K, Culleton BF, Manns BJ, et al. NSAID use and progression of chronic kidney disease. Am J Med. 2007;120(3):280 e281-287. doi:10.1016/j.amjmed.2006.02.015

46. Ungprasert P, Cheungpasitporn W, Crowson CS, Matteson EL. Individual non-steroidal anti-inflammatory drugs and risk of acute kidney injury: a systematic review and meta-analysis of observational studies. Eur J Intern Med. 2015;26(4):285-291. doi:10.1016/j.ejim. 2015.03.008

47. Manappallil RG, Prasan D, Peringat J, Biju IK. Severe bone marrow suppression due to methotrexate toxicity following aceclofenac-induced acute kidney injury. BMJ Case Rep. 2018; 2018:bcr-2018-224722.

48. Geenen R, Overman CL, Christensen R, et al. EULAR recommendations for the health professional's approach to pain management in inflammatory arthritis and osteoarthritis. Ann Rheum Dis. 2018;77 (6):797-807.

49. Qaseem A, Wilt TJ, McLean RM, Forciea MA; Clinical Guidelines Committee of the American College of P. Noninvasive treatments for acute, subacute, and chronic low back pain: a clinical practice guideline from the American College of Physicians. Ann Intern Med. 2017;166(7):514-530. doi:10.7326/ M16-2367

50. Kolasinski SL, Neogi T, Hochberg MC, et al. 2019 American College of Rheumatology/Arthritis foundation guideline for the management of osteoarthritis of the hand, hip, and knee. Arthritis Rheumatol. 2020;2:220-233.

51. van der Heijde D, Ramiro S, Landewe R, et al. 2016 update of the ASAS-EULAR management recommendations for axial spondyloarthritis. Ann Rheum Dis. 2017;76(6):978-991.

52. Ward MM, Deodhar A, Gensler LS, et al. 2019 Update of the American College of Rheumatology/Spondylitis Association of America/Spondyloarthritis research and treatment network recommendations for the treatment of ankylosing spondylitis and nonradiographic axial spondyloarthritis. Arthritis Rheumatol. 2019;71 (10):1599-1613. doi:10.1002/art.41042

53. Perís F, Martinez E, Badia X, Brosa M. Iatrogenic cost factors incorporating mild and moderate adverse events in the economic comparison of aceclofenac and other NSAIDs. Pharmacoeconomics. 2001;19 (7):779-790. doi:10.2165/00019053-200119070-00006

54. Gijón Baños J. [Efficacy and safety of nabumetone in the treatment of knee osteoarthritis: a comparative clinical trial versus aceclofenac. Study Group of Nabumetone for Osteoarthritis of the Knee]. Med Clin. 1997;109(4):130-134. Italian.

55. Jagadeesh P, Devi BV, Vijayalakshmi P. A study on efficacy and safety of nabumetone and aceclofenac in osteoarthritis patients using visual analogue scale. IOSR Journal of Dental and Medical Sciences. 2018;17 (4):40-45. doi:10.9790/0853-1704084045 


\section{Publish your work in this journal}

The Journal of Pain Research is an international, peer reviewed, open access, online journal that welcomes laboratory and clinical findings in the fields of pain research and the prevention and management of pain. Original research, reviews, symposium reports, hypothesis formation and commentaries are all considered for publication. The manuscript management system is completely online and includes a very quick and fair peer-review system, which is all easy to use. Visit http:// www.dovepress.com/testimonials.php to read real quotes from published authors.

Submit your manuscript here: https://www.dovepress.com/journal-of-pain-research-journal 